Man and Nature

L'homme et la nature

\title{
Répétition et instabilité : la signification de Zadig
}

\section{Roseann Runte}

Volume 3, 1984

URI : https://id.erudit.org/iderudit/1011825ar

DOI : https://doi.org/10.7202/1011825ar

Aller au sommaire du numéro

Éditeur(s)

Canadian Society for Eighteenth-Century Studies / Société canadienne d'étude du dix-huitième siècle

ISSN

0824-3298 (imprimé)

1927-8810 (numérique)

Découvrir la revue

Citer cet article

Runte, R. (1984). Répétition et instabilité : la signification de Zadig. Man and

Nature / L'homme et la nature, 3, 63-76. https://doi.org/10.7202/1011825ar

Copyright (c) Canadian Society for Eighteenth-Century Studies / Sociéte canadienne d'étude du dix-huitième siècle, 1984
Ce document est protégé par la loi sur le droit d'auteur. L'utilisation des services d'Érudit (y compris la reproduction) est assujettie à sa politique d'utilisation que vous pouvez consulter en ligne.

https://apropos.erudit.org/fr/usagers/politique-dutilisation/ 


\section{Répétition et instabilité: la signification de Zadig}

Dans l'introduction à son Essai sur Candide, Jean Sareil nous signale qu'aussi paradoxal que cela puisse paraître, deux siècles d'une abondante exégèse n'ont pas réussi à clarifier la signification d'un conte qui se veut naïf et simple. Les nombreuses interprétations de Zadig permettent de dire autant de ce conte. Il est donc avec beaucoup d'humilité que nous présentons ces quelques pensées sur Zadig.

La question centrale à tous les débats sur Zadig peut être réduite à un seul mot: 'Mais.' Faut-il prendre Voltaire au sérieux et croire que la leçon du conte est que nous sommes tous les marionnettes de la Providence et que nous devrions nous résigner à notre destin inaltérable? Ou faut-il plutôt croire que Voltaire se moque de l'idée exprimée par Pope et Leibnitz et selon laquelle chaque événement fait partie de l'ordre de l'univers, et selon laquelle tout mal entraîne un plus grand bien? Ou est-ce que le conte illustre le dilemme de Voltaire qui doit reconnaître l'existence ou la co-existence du mal et du bien sans pouvoir concevoir comment ils s'accordent?

Pour soutenir ces théories, certains critiques se sont servis de preuves d'ordre biographique et ont cité alternativement les prétendues réussites et les déceptions de l'auteur à l'époque où il rédigeait le conte. D'autres critiques se sont efforcés de montrer par le développement ou le nondéveloppement du personnage principal, par la structure ou par l'absence de structure, par l'unité ou la dualité du complot, par l'ironie ou par son absence, que l'une ou l'autre des trois interprétations évoquées l'emporte. 
Il y a cependant un grand mal dont souffrent unanimement toutes ces interprétations: aucune n'est assez compréhensive pour éviter ou pour expliquer les contradictions apparentes que nous nous permettrons de relever brièvement.

Van den Heuvel traite Zadig en Bildungsroman. ${ }^{1}$ Selon lui, Voltaire montre de plus en plus de sympathie pour ses personnages qui souffrent et évoluent. Zadig souffre, il est vrai, et il est également vrai qu'il est et roi et heureux à la fin. Mais le bonheur, comme cette mise en parallèle d'un substantif et d'un adjectif quasiment antithétiques, est instable. Nous constatons que chaque fois que Zadig se croit heureux, il est sur le point de devenir malheureux. Zadig, s'il a appris une seule chose, a appris à redouter les serpents lorsqu'il jouit d'un lit de roses. Plutôt qu'un Zadig heureux, nous voyons un Zadig prêt à retomber dans le malheur. Le lecteur est, pour ainsi dire, conditionné, après dix-neuf leçons, à ne plus s'en étonner. ... D'ailleurs, si Zadig doit son bonheur, comme il le dit, aux leçons qu'il a retenues: celle de l'ermite devenu ange et celle du voleur devenu riche, il est guidé par des idées contradictoires: on doit se soumettre au destin et on ne doit pas l'accepter. Si telle est donc la sagesse de Zadig, il est difficile de croire que l'élève est l'objet de la sympathie de l'auteur. N'oublions pas non plus que Zadig a énormément pitié de luimême: il se croit le plus malheureux des hommes, au point de s'estimer, séparé qu'il est de son amour, cent fois plus malheureux que ce pêcheur qu'il rencontre et qui a perdu sa femme, sa maison, son commerce et qui est un homme tellement pitoyable que même les poissons (comme les hommes d'ailleurs) se moquent de lui. Chose bizarre, Zadig nous donne toujours la liste de ses malheurs, mais oublie de mentionner les bonnes choses ou n'en compose simplement pas la liste lorsqu'il est heureux. Comme Pangloss, il répète sa liste de malheurs et attribue mal les effets aux causes. Mais Pangloss est convaincu de sa philosophie et rien ne l'ébranle. En revanche, Zadig n'a que sa foi religieuse pour le soutenir, et ses malheurs et son manque de compréhension font qu'il murmure contre son destin apparent et contre la Providence. Mais revenons à la question de sympathie: cet homme qui dort si bien et si tard qu'il se fait voler, qui refuse de suivre les conseils de son ami, qui est si inefficace, si incapable d'agir et si prompt à se plaindre, cet homme est plutôt l'objet de notre pitié.

Il est également difficile de croire, avec Roland Barthes, à un Zadig idéalement bon du début à la fin. ${ }^{2}$ Très tôt Voltaire nous dit de Zadig: 'Il était aussi sage qu'on peut l'être: car il cherchait à vivre avec des sages. ${ }^{\prime 3}$ La sagesse n'est pas une maladie contagieuse; on peut vivre longtemps avec des sages et rester parfaitement sot. D'autre part, qui sont et où sont ces prétendus sages? La compagnie de Zadig se compose de vaniteux, 
d'ingrats, d'infidèles, de jaloux et d'envieux: Sémire et Orcan, par exemple. Voltaire nous dit également que Zadig ' $n$ 'ignorait pas les principes physiques de la nature, tels qu'on les connaissait alors, et savait de la métaphysique ce qu'on en a su dans tous les âges, c'est-à-dire fort peu de chose' (p. 3). Zadig avait aussi 'de grandes richessess' et 'par conséquent, des amis' (p. 3). Il nous semble que Zadig manque d'amis, de métaphysique, de sagesse et même d'amour, passion qu'il ne découvrira qu'avec la jarretière de la femme de l'envieux et les rubans jaunes de la reine. (Les symboles de l'amour et de la jalousie sont interchangeables.) Si Zadig parle plusieurs langues et fait preuve d'une bonne interprétation de la justice, il est tout de mème totalement superficiel dans son raisonnement et complètement aveugle quant à ses rapports avec autrui. Zadig l'imparfait philosophe admet d'ailleurs qu'il ne comprend pas le discours des autres philosophes. Il ne se doute nullement des actions des jaloux et des envieux. Il murmure contre la même Providence qu'il nie. Enfin, il ne semble ni idéalement bon ni parfaitement sage.

Zadig n'est cependant pas totalement idiot comme l'ont suggéré d'autres critiques. ${ }^{4}$ Il est toujours celui des hommes qui mérite le plus d'être éclairé, ce qui n'est pas très flatteur pour le lecteur.

Nancy Senior divise notre conte en deux: l'histoire du héros parfait, celui qu'épouse Astarté, et l'histoire du philosophe qui commence par questionner timidement et qui finit par tout questionner, même le discours de l'ange Jesrad. ${ }^{5}$ Mais Senior admet que la solution n'est guère parfaite, car il y a des épisodes qui ne contribuent ni au développement de Zadig le sage, ni à celui de Zadig l'amoureux. Ces épisodes sont, conclut-elle, tout simplement amusants. Mais cet amoureux parfait ne tombe amoureux qu'au beau milieu du conte et ne se rappelle son amour qu'à la fin des chapitres, au moment où, après une autre aventure, il cherche l'Astarté de ses rêves sans pourtant la reconnaître lorsqu'il la retrouve. L'amoureux parfait n'est guère touché par Almona qui lui sauve la vie et lui offre son coeur. Il préfère voler la femme d'un autre, tout comme Orcan lui a pris Sémire. On pourrait d'ailleurs se demander s'il y a de l'amour ou seulement de la jalousie dans ce conte.

Zadig ne ressemble pas non plus à un philosophe parfait. Il se prosterne à l'envolée de l'ange et sa pire hérésie est de 'murmurer contre la Providence' qu'il finit par adorer (répété deux fois) et bénir (le dernier mot) (p. 59). Et si Zadig se permet de murmurer contre la Providence, c'est dans un état de choc ou de dépression, non pas à la suite d'un discours ou d'un soliloque philosophique, chose à laquelle il ne participe qu'une fois (lors du souper) et dont la conclusion (la Providence existe) ne nous révèle pas si cette Providence est bonne ou mauvaise.

Mais est-ce que le conte nous apprend que nous sommes libres ou que 
nous sommes victimes de notre destin? La réponse se trouve, presque tous les critiques le disent, dans le chapitre sur l'ermite. ${ }^{6}$ L'ange donne à Zadig la triple illustration du fait qu'il n'y a point de hasard. Nous voudrions bien le croire, mais ... . Examinons d'abord de plus près ce chapitre.

Selon les dires de l'ermite, il n'y a donc point de hasard. Zadig est plus ou moins convaincu par les deux premières illustrations de l'ermite, mais il se révolte contre le troisième exemple. Il proteste contre l'apparente cruauté de l'ermite qui soutient que ses crimes ont prévenu de plus grands malheurs. Zadig objecte trois fois et refuse d'accepter aussi bien la nécessité que l'apparente universalité et l'injustice du mal. L'ermite répond mal, avec des arguments circulaires: le mal existe partout sauf au Paradis; comme la terre n'est pas le Paradis, il y a nécessairement du mal ... . A la dernière intervention de Zadig, l'ermite devenu ange et équipé d'ailes, s'envole. Zadig s'agenouille, adore et proteste en prononçant un dernier 'mais' (p. 57). L'ange dit ensuite à Zadig d'aller à Babylone. Zadig ne suit pas ce conseil et marche plutôt au hasard. En dépit du hasard, ou peut-être à cause de lui, il arrive à Babylone. Voltaire le rusé joue avec son lecteur, il fait même de notre dilemme un jeu de mots!

Chez Voltaire, le mot 'adorer' endosse, Van den Heuvel nous le signale, une signification ironique, et l'envolée de l'ange nous fait penser au derviche dans Candide, qui fermera la porte au nez du héros interrogateur; elle nous fait penser également au Sirien dans Micromégas qui s'envole, lui aussi et laisse un beau livre illisible pour l'Académie des Sciences.' Cependant Zadig s'agenouille et finit par entrer dans la ville de Babylone. Peut-être qu'il accepte, en murmurant, la notion de destin. On peut aussi croire que Zadig agenouillé n'est ni debout ni aplati, les bras étendus en forme de croix (comme ses ancêtres médiévaux l'étaient), et qu'il n'accepte ni ne rejette le destin, qu'il est en proie à des doutes. Nous ne trouvons la clef au reste du conte ni dans ce chapitre ni à la fin où Zadig pense au grain de sable (il doute) et où il adore et bénit la Providence dans une seule phrase qui résout tout le conte et rien.

Si l'on considère le destin comme le thème central du conte, il y a beaucoup d'exemples d'hommes qui échappent à leur destin, notamment le héros, Zadig, qui devait mourir au moins trois fois, mais qui s'est échappé grâce à un mauvais médecin, un ami et un billet doux livré très à propos, entre la poire et le poison, pour ainsi dire. Zadig intervient et change le destin du pêcheur, et même le sien propre. Dans beaucoup de chapitres on ne parle nullement du destin, mais plutôt de l'infidélité des femmes, des hommes, de l'inaptitude des médecins, de la corruption des juges, etc.

Le destin et le hasard sont, certes, des thèmes importants dans Zadig, 
mais sont-ils des thèmes centraux? Voltaire lui-même ne nous prouve-t-il pas que le hasard à la fois existe et n'existe pas? Si l'on divise le conte d'après le thème du hasard, les chapitres se groupent en quatre catégories: 1 ) le destin ne rémunère pas la bonté; 2 ) le destin rémunère la bonté; 3) le destin rémunère le mal; 4) le destin peut rémunérer la bonté. Ces groupes s'opposent symétriquement et se réduisent par là à rien en termes de signification. La structure du conte, de ce point de vue, ressemble au discours de Zadig qui dit une chose ('mais') et en fait une autre (il s'agenouille), et à celui du narrateur qui le laisse s'égarer, mais dans la direction de Babylone. Si l'on poursuit ce thème on devra conclure comme le fait le narrateur de l'Histoire du Bon Bramin: 'il y a là de quoi parler beaucoup' (p. 116). Cette interprétation ressemble à celles de René Pomeau et de Lester Crocker. Selon eux, Voltaire nous explique un dilemme, mais n'y voit pas de solution lui-même. ${ }^{8}$ Nous ne voudrions cependant pas accepter un 'non liquet' comme étant la principale signification de notre conte.

Aussi avons-nous pensé à donner à Zadig un sous-titre: 'ou la recherche du bonheur.' Zadig commence sa quête d'une manière assez systématique: il cherche le bonheur chez les femmes (nobles et citoyennes), à la campagne, à la cour, dans les sciences, chez les savants et les honnêtes gens. Mais après le quatrième chapitre il s'arrête. Il oublie apparemment qu'il cherche le bonheur - il se croit d'abord heureux et est ensuite obligé de chercher Astarté. La quête d'Astarté remplace ainsi la quête du bonheur. Astarté représente non seulement la passion mais aussi le pouvoir. Sans elle, Zadig se croit le plus malheureux des hommes - mais il se trompe, et nous le savons. D'une part, le pêcheur semble bien plus à plaindre que Zadig, même si celui-ci nous assure que les malheurs du coeur sont les plus graves. D'autre part, le narrateur nous a déjà appris que la lune de l'absinthe suit la lune de miel. Le bonheur est instable, indéfinissable et trompeur - il ne faut pas croire aux apparences.

Nous avons alors imaginé un deuxième sous-titre dans l'espoir de découvrir le lien secret entre tous les chapitres du conte: 'ou l'amitié.' En effet, le thème de l'amitié parcourt l'ensemble du conte. D'abord, Zadig a des amis uniquement à cause de ses richesses. Ensuite, il paie Cador pour s'assurer de son amitié. Plus tard il envoie le pêcheur chez Cador, car il semble avoir enfin appris que l'amitié dépasse les considérations monétaires. Et Zadig serait à la fin le seul monarque sur terre qui eût un ami.

Mais Zadig semble toujours préférer la passion à l'amitié, et pourtant il est continuellement sauvé par ses amis. S'il n'est pas victime du destin, c'est grâce à ses amis qui arrivent à en contrecarrer les coups. Zadig 
rouspète sans cesse contre son mauvais destin, mais lorsque ses amis le tirent d'affaire, il loue la Providence. Il ne se montre que rarement reconnaissant. Cador ressemble à un sage, peut-être à Tiberge, l'ami de Des Grieux dans Manon Lescaut de Prévost. Il donne de bons conseils à Zadig qui ne les suit pas. Il avertit Zadig du danger et ce dernier refuse d'y faire attention. Cador le sauve: des prêtres, des juges, des envieux, des jaloux. Il est capable de sauver Zadig de tout sauf de la passion qui dévore notre héros. (Et il ne faut pas oublier que le narrateur condamne cette passion et la traite comme une maladie: 'Zadig fut longtemps malade, mais enfin la raison l'emporta sur son affliction...' [p. 4].) Au début Zadig a pour Sémire un attachement solide et vertueux tandis qu'elle l'aime avec passion. La passion se montre fragile et ne dure qu'aussi longtemps que Zadig garde sa beauté physique. Par la suite, presque toutes ses mésaventures sont causées soit par la passion des autres et souvent celle des femmes (comme celle de l'envieux), soit par sa propre passion qui le rend aveugle - qui ne lui permet pas de raisonner ni de reconnaître ses amis. Zadig, en ami raisonnable, sauve la vie à Almona qui est en proie à la passion du paraître. A son tour, Zadig est sauvé par Almona et pour la première fois il montre de la reconnaissance - il se jette à ses pieds. Il aide ensuite le malheureux laitier devenu pêcheur en lui disant d'aller voir Cador. Zadig reconnaît, du moins indirectement, l'amitié et le fait qu'il pourra toujours compter sur Cador. Et, détail intéressant à noter, quand le pêcheur veut montrer sa reconnaissance, il se jette aux pieds de ''ami de Cador' (p. 41). Le narrateur, pour la première fois, désigne Zadig autrement que par son nom. Zadig est enfin devenu ami et, deux chapitres plus loin, il voit clair et distribue sagement des bénéfices à ses amis. Zadig endosse finalement les caractéristiques de son ami raisonnable. Mais cette fin est un tant soit peu féerique car elle combine pouvoir, amour et amitié, mélange instable, sinon impossible. D'ailleurs, si le thème du conte est vraiment l'amitié, plusieurs chapitres tels que 'L'Ermite,' 'Le Souper' et 'La Femme battue,' sont superflus. L'amitié doit alors être considérée comme un sous-thème, un leitmotif, et nous devons de nouveau nous mettre à la recherche du thème principal de Zadig.

Voltaire a fait, au début du conte, des promesses auxquelles notre naïveté nous permettra de croire. Il nous a averti de ce que son conte est 'un ouvrage qui dit plus qu'il ne semble dire' (p. 1). En comparant Zadig aux histoires des Mille et une Nuits, le sage Oulong note que ces dernières 'sont sans raison et ne signifient rien' (p. 2), ce qui permet d'inférer que Zadig a une raison et signifie quelque chose de plus qu'il ne semble signifier à première vue.

En cherchant une signification qui donnerait de l'unité au conte, nous 
nous sommes rendu compte du fait que tout est contredit ou contradiction dans Zadig. Si Zadig se jette à genoux devant l'ermite devenu ange, il se prosterne aussi devant les chandelles avec lesquelles il illustre le ridicule de l'adoration. Si Zadig appelle l'ermite 'un ange sauveur,' le pêcheur donne ce même titre à Zadig. Si Zadig est victime des passions coupables de sa femme, il inspire ces mêmes sentiments à la femme d'un autre. S'il est rendu malheureux par l'infidélité des êtres humains, il est aussi sauvé par cette même infidélité. Zadig est esclave et roi, victime et vainqueur. Chaque épisode a un parallèle qui semble donner une leçon contradictoire. Chaque geste et chaque symbole est suivi de son antithèse. Souvent ces antithèses sont introduites par un 'mais.' Le mot-clé du chapitre de l'ermite est répété dix fois dans ce seul chapitre et au moins cinquante-sept fois dans le reste du récit. ${ }^{9}$ 'Mais' présente, par définition, une contradiction, et Voltaire s'en sert pour créer des contradictions même là où l'on ne s'attend pas à en trouver. Il emploie l'antithèse synonymique, procédé que Deloffre ${ }^{10}$ a relevé chez Marivaux. Dans notre texte nous trouvons les exemples suivants: 'Zadig n'était point un assassin ... mais il était coupable du sang d'un homme' (p. 26); 'je n'étais point coupable mais ... je vais mourir criminelle' (p. 22). Ces 'mais' ajoutent à l'effet comique créé par le caractère inattendu de la phrase qui suit. ${ }^{11}$ Dans l'exemple suivant nous nous attendons, après la boule de neige linguistique de toutes les possibilités d'agir, à une action: 'Zadig fut tenté à la fois d'éclater de rire, de dire des injures au révérend père, de le battre, et de s'enfuir mais ... il ne fit rien de tout cela' (p. 55). Zadig est parsemé de 'mais,' mot qui devient, avec la litanie des malheurs de Zadig, un refrain dont l'ironie a déjà été signalée dans les études de Topazio et Van den Heuvel. Ceux-ci citent l'emploi du 'mais' dans d'autres textes de Voltaire tels que la lettre à Madame Denis: Voltaire lui y écrit que les soupers du roi Frédéric II sont délicieux ... mais. Le 'mais' clot les trois débats (ou mini-débats) philosophiques de Zadig: le souper, l'esclavage et l'ermite. Les trois conclusions se contredisent mutuellement et contredisent au moins une partie du débat à l'intérieur de la discussion. Un véritable crescendo de 'mais' accompagne le thème secondaire du bonheur instable.

Après seulement quatre chapitres, Zadig croit à la possibilité du bonheur. Le narrateur intervient pour nous informer que Zadig se trompe. Quelques chapitres plus loin, Zadig pense qu'il est enfin heureux, mais il commence lui-même à se douter de l'instabilité du bonheur. Il demande alors au perroquet du roi si le bonheur sera de courte durée. La question est posée d'une manière qui indique le pessimisme naissant de Zadig. L'oiseau répond affirmativement et le prochain malheur s'annonce déjà. Enfin, Zadig se dit, en songe, bien entendu 
(il parle aux oiseaux, mais il ne se parle pas), que son bonheur va bientôt s'évanouir. Le narrateur, l'oiseau et Zadig ont tous eu raison, chacun à son tour. Alors, quand à la fin du conte nous apprenons que Zadig fut roi et fut heureux, la phrase nous choque par sa qualité insolite. Après dixneuf leçons et une centaine de pages d'exemples, le lecteur a envie d'ajouter: 'mais où est le serpent?' Et il $\mathrm{y}$ a beaucoup de serpents potentiels. Zadig est heureux grâce à sa femme. Mais il a déjà été trompé par ses deux premières épouses. Nous avons appris que la lune de miel est suivie de la lune de l'absinthe. Nous nous souvenons du mariage de Sétoc: 'Sétoc devait aller ... dans lîle de Serendib; mais le premier mois de son mariage qui est, comme on le sait, la lune de miel, ne lui permettait ni de quitter sa femme, ni de croire qu'il pût jamais la quitter' (p. 60). Nous nous rappelons Zadig qui a répudié Azora quelque temps après le second mois (p. 6). Et nous avons sous les yeux le sage exemple du pêcheur qui, lui, prend l'argent et refuse la femme (p. 59). Même dans l'exemple de Sétoc qui n'a pas encore quitté sa femme, la phrase est négative et suggère que le bonheur ne va pas durer. Il est donc naturel de penser à la lune de l'absinthe quand enfin Zadig épouse Astarté. Voltaire décrit cette dernière scène avec des superlatifs: 'ce fut le plus beau siècle de la terre' (p. 59). Nous avons déjà assez souvent entendu que Zadig fut 'le plus malheureux des hommes' (p. 41, par exemple). Si nous rejetons la première exagération, nous en ferons autant des autres. Nous ne croirons pas au véritable bonheur de Zadig et nous ajouterons nous-mêmes la phrase prophétique: 'mais il se trompe.'

Tout est donc contradictoire et les apparences sont trompeuses. Le conte, selon Voltaire, dit plus qu'il ne semble dire. Les synonymes se transforment en antonymes. Le grand débat dans le chapitre du 'Souper' n'est pas un débat du tout, car Zadig, même s'il n'a pas tout compris, a assez compris pour savoir que tous les interlocuteurs partagent le même avis. Zadig montre à Sétoc le ridicule de ses croyances en l'imitant avec exagération. L'ange montre à Zadig que ce qui paraît bon peut être mauvais et vice versa.

Zadig attribue constamment ses malheurs à des raisons superficielles. Il croit, par exemple, avoir perdu la reine et le royaume parce qu'il a fait la grasse matinée et non parce qu'il a des ennemis motivés par l'envie et la jalousie.

Tout le mal qui arrive à Zadig est causé par les passions des hommes. Ce n'est pas le destin qui le condamne à mort, mais les hommes - les hommes avec leur envie de l'argent et du pouvoir. Zadig est incapable de comprendre les femmes qu'il juge par leur beauté et par la classe sociale. Les femmes ne sont guère meilleures que les hommes et les quittent quand ils deviennent laids ou sentent l'approche de la mort. Les femmes, tout 
comme les hommes, sont elles aussi influencées par le paraître. Zadig est un habile juge qui sait résoudre les problèmes des autres, mais il est incapable d'identifier ses propres ennemis. Quand on loue son sens de la justice, il ne comprend pas que l'on le flatte et que l'on ne l'aime que pour sa position dans le gouvernement. Même la veuve Almona est victime des apparences: elle détestait son mari mais elle se brûlera pour sauver les apparences: 'Tout le monde se moquerait de moi si je ne me brûlais pas' (p. 30). Et les juges corrompus sont condamnés moins pour avoir aimé cette femme que pour avoir été hypocrites.

Les méchants semblent heureux et les heureux, méchants. Le malheur même est mis en question. Nous assistons à un concours de malheurs entre Zadig et le pêcheur, concours à la fin duquel Zadig se déclare le gagnant car son malheur invisible est pire qu'un malheur visible. Zadig gagne le combat contre Itobad, mais il n'est pas reconnu vainqueur à cause, encore une fois, des apparences. Itobad revêt l'armure blanche de Zadig et les juges et le public vont couronner non le vainqueur mais ses vêtements. Ils permettent à Zadig de se présenter pour expliquer les énigmes, non à cause de sa probité, mais à cause de sa réputation de probité. Astarté n'est pas simplement heureuse d'être réunie avec Zadig, mais elle 'goûtait ... la douceur de voir son amant digne aux yeux de l'univers d'être son époux' (p. 59). Zadig devient roi, époux et heureux. Il règle ses dettes, punit ses ennemis, et le lecteur attend la prochaine mésaventure car il a bien appris qu'il ne faut pas juger quoi que ce soit, que tout est trompeur, que tout est instable.

Une comparaison de Zadig et de Candide révèle une différence d'ordre structural. Candide n'est jamais seul. Il est toujours accompagné de Pangloss ou de Martin. Il y a donc possibilité de dialogue. Candide peut essuyer des mésaventures et murmurer contre son destin tout en écoutant une leçon d'optimisme. Il existe une certaine tension entre les personnages et Candide a le choix d'apprendre la leçon qu'il tirera des événements ou des dires de ses amis. Zadig, au contraire, est seul et doit agir et énoncer la leçon en même temps. Il n'est pas parfaitement sage, ni au début ni à la fin. Il est cependant obligé de nous résumer sa propre leçon avant de l'avoir apprise. Il ne peut certainement pas nous dire ce qu'il ne sait pas, et Voltaire ne peut pas faire intervenir trop souvent des oiseaux parlants oú faire parler notre héros en songe. Or, c'est en faisant de Zadig un mauvais élève que Voltaire pourra exprimer ses idées. Zadig dira le contraire de ce que Voltaire veut dire, ou il se taira. Voltaire nous signale que tel est le rôle des 'mais.' Rien n'est ce qu'il semble être.

Le conte est doublement ironique, car Zadig répète une litanie de malheurs qui est instable. Les malheurs inscrits sur la liste changent continuellement. Seule reste constante l'ironie de la contradiction: Zadig, le 
plus intelligent des hommes, tire toujours la mauvaise conclusion des leçons et fait des erreurs de logique aussi évidentes que l'est l'ironie.

Voltaire n'a pas inventé l'histoire du nez; il n'a pas inventé l'histoire de l'ermite non plus. Ce commentaire n'a rien d'original: Fréron accusa déjà Voltaire de plagiat il y a deux siècles. Parmi les sources possibles figurent de vieilles légendes hébraïques, le Coran, les Vitae patrum, les Gesta Romanorum, le Libro de los enxemplos, les Exempla de Jacques de Vitry, De causa dei de Bradwardine, Luther, Antoinette Bourignon, Herbert, Jean le Jeune, Henry More, Cotton Mather et le poème The Hermit de Parnell.12 L'histoire de l'ermite est un renversement complet du texte médiéval français, texte que Voltaire a sans doute connu et utilisé, comme il a utilisé celui de Parnell.

Le texte médiéval a pour but de calmer les esprits d'un ermite qui a commencé à douter d'une Providence qui semble punir les bons et enrichir les méchants. La leçon que l'ange lui donne est celle de la résignation à la Providence, car l'apparente injustice terrestre sera corrigée dans une autre vie où règnera la grâce. Les exemples, aussi illogiques qu'ils semblent, servent à rassurer l'ermite qui se tait devant le discours de l'ange et qui retourne humblement à sa cave pour prêcher la gloire de Dieu. Le conte illustre l'idée exprimée par Saint Thomas d'Aquin et selon laquelle les actes de violence inexplicables sont en eux-mêmes la démonstration de l'existence de Dieu. La violence de l'ange sauve l'âme du pêcheur en puissance, et la vie de la victime potentielle n'est qu'un dérivé accidentel.

Dans la version de Voltaire les mêmes épisodes qui calment le doute de l'ermite inspirent le doute à Zadig. Voltaire remplace le règne de la grâce par le règne de la justice. Il n'est plus question d'une vie dans l'Au-delà. Tout se passe sur terre - la gloire est terrestre. Il est vrai que Zadig ne peut pas lire dans le livre des destins, mais il sait résoudre des énigmes. Dans le chapitre 'Le Brigand,' Voltaire présente l'idée qui choque tant Zadig qu'il ne dort pas de la nuit: 'L'empire est déchiré, et ce brigand est heureux: ô fortune! ô destinée! un voleur est heureux et ce que la nature a fait de plus aimable a péri peut-être d'une manière affreuse' (p. 38). Voltaire reproduit ensuite une histoire qui 'prouve' que la justice existe après la mort, et qui met en doute le rôle de la Providence: Zadig parle et l'ange, muet, s'envole. Dans la version médiévale, c'est l'ange qui parle et l'être humain ne dit mot. En réalisant les rêves de Zadig à la fin du conte, Voltaire est très ironique: ou bien il veut nous faire croire à un autre exemple de l'illogique du système du bien et du mal (pourquoi faire gagner Zadig maintenant?), ou bien la leçon du conte est une négation de l'éternel qui est ainsi transitoire et terrestre.

Dans Zadig les méchants paraissent heureux et souvent innocents, et 
les bons, malheureux et coupables. Toute récompense est uniquement terrestre et limitée au présent. Les récompenses ne sont cependant ni visibles ni compréhensibles. C'est une vue extrêmement pessimiste. Voltaire n'offre aucun remède. L'homme est entièrement incapable de juger, car comment juger autrement que selon les apparences? Zadig aide la femme battue une première fois car elle semble souffrir, mais il apprend qu'elle aime être battue. La deuxième fois Zadig refuse de l'aider car il ne croit plus aux apparences. Nous ne saurons jamais sil avait raison, mais habitués à l'ironie, nous sommes portés à croire qu'elle souffrait véritablement la deuxième fois. ${ }^{13}$

Zadig est composé d'un nombre d'histoires dont trois sont puisées dans l'histoire littéraire et intercalées dans le reste du récit: l'histoire du nez, celle du bûcher et celle du pêcheur. Chacune de ces histoires est précédée d'une introduction (ou pré-histoire) ou suivie d'une conclusion (ou post-histoire) qui souligne la leçon de l'histoire empruntée. L'histoire du 'Borgne' précède celle du 'Nez.' Dans les deux cas, Zadig faillit perdre une partie de son anatomie, et perd soit sa femme soit sa fiancée. Dans 'Le Borgne,' la femme qui semble aimer n'aime que les apparences. Elle n'aimera pas un Zadig borgne. Dans 'Le Nez,' Zadig essaie d'apprendre à cette femme de ne pas juger selon les apparences. Il se trompe et apprend, à son malheur, que sa femme ne l'aime pas. Zadig lui aussi juge selon les apparences et attribue le mal d'abord à la classe sociale de sa fiancée et ensuite à toutes les femmes sans penser que le mal vient de la nature passionnée de sa fiancée dans la première histoire et de sa propre passion dans la deuxième. Azora est raisonnable et logique. Zadig, mort, n'aura vraisemblablement pas besoin de son nez. Zadig, motivé par la vengeance, avait construit le piège dans lequel il a fini par attraper sa femme et perdre, par conséquent, son propre bonheur.

Nous apprenons dans 'Le Bûcher' et 'Les Rendez-vous' que la société juge du paraître plutôt que de l'être et que le résultat est le règne de l'injustice. 'L'Ermite' et 'Le Pêcheur' nous enseignent qu'il est impossible de croire à un système divin de récompenses et de punitions, car les récompenses et les punitions sont indistinguibles. Les personnages victimes des événements ne savent même pas s'ils sont vraiment malheureux.

La gradation que nous observons dans les trois paires d'histoires va de l'individu à la société au surnaturel. Personne ne sait distinguer l'être du paraître. Selon la leçon qui unit ces histoires, il faut examiner d'un œil critique l'image que l'on projette autant que celle que l'on perçoit.

Le 'mais' constitue l'objection rationnelle à la démonstration surnaturelle. C'est le doute qui est inspiré par ce qui était à une époque révolue l'évidence irréfutable de la Providence. En général, le 'mais' con- 
stitue un leitmotif qui sert à nous avertir de ce que le terrain est instable et changera d'aspect avec notre point de vue.

Ainsi averti, le lecteur a la satisfaction d'être mieux informé que le public envisagé dans le texte. Il ne croit pas qu'Itobad ait vaincu Zadig. Il ne pense pas, comme le pêcheur, que Zadig soit un ange sauveur, et il ne sera certainement pas coupable d'avoir jugé quelqu'un par la couleur de ses jarretières.

Il y a donc complicité entre le narrateur et son lecteur. Cette complicité est exprimée sous forme de dialogue. Le narrateur communique son opinion dans les brefs commentaires qu'il ajoute à la narration. En voici un exemple: 'Zadig disait: "Je suis donc enfin heureux!" Mais il se trompait' (p. 15). L'opinion tranchante du narrateur est communiquée dans un style nu et presque télégraphique. L'oiseau, lui aussi, transmet l'opinion du narrateur et Zadig la réitère en rêvant. Le 'mais' sert également de rappel constant à la réalité du narrateur. Le lecteur ne doit rien accepter comme acquis. Des événements au vocabulaire, tout se contredit. Voltaire emploie le même procédé dans tous ses contes. Le lecteur est systématiquement éduqué à raisonner et à ne rien croire. Les morts se raniment. Zadig guérit en dépit des médecins. Il est injustement condamné à l'esclavage par les Egyptiens qui 'étaient alors justes et humains' (p. 26). Il est injustement condamné à mort par un bon roi qui 'crut tout ce qu'il voyait, et imagina tout ce qu'il ne voyait point' (p. 21). Même les descriptions sont trompeuses. Comme l'a noté Jean Sareil, Almona avait un nez 'qui n'était pas comme la tour du mont Liban' (p. 35), c'est-à-dire, qui était probablement petit. ${ }^{14}$

Le narrateur communique avec son lecteur, et lorsque celui-ci s'arrête et relit ce texte stylistiquement trompeur, le dialogue s'installe. Il y a aussi dialogue lorsque le lecteur lit, 'Zadig fut roi, et fut heureux' (p. 59), et substitue le mot 'mais.' Il y a enfin dialogue quand le lecteur, arrivé à la dernière page, est prêt à continuer et tourne la page dans la ferme conviction que le règne de la gloire, de l'abondance, de la justice et de l'amour sera suivi d'une nouvelle mésaventure. Le narrateur est étrangement absent dans le dernier paragraphe. Il n'y a pas un seul 'mais.' Le nain semble avoir renoncé à la peinture et le perroquet semble avoir été oublié. Et Zadig, qui ne prononce pas un seul'mot, semble avoir bien retenu ses leçons. Mais quelles leçons? Celle de l'ange (on ne peut pas échapper à sa destinée) et celle du brigand (on peut changer son destin par ses propres efforts). Si le lecteur n'a pas déjà dit, 'mais quel sera le serpent,' il fera comme Zadig devant l'ermite: il demandera 'la permission de parler' (p. 56) et il objectera, comme Zadig: 'Mais ... .' 'Mais ... s'il n'y avait que du bien et point de mal' (p. 56)? L'ermite a déjà répondu: 'Alors, ... cette terre serait une autre terre' (p. 56). Voltaire en donne l'illustration con- 
crète avec cette fin féerique. De plus, un subtile changement s'opère. A la place d'un Zadig qui demande une réponse à l'ermite devenu ange, Voltaire substitue le lecteur. Celui-ci pose des questions au narrateur qui s'absente tout comme l'ange s'envole. Il est ironique que ces questions sont celles que le narrateur a déjà posées au lecteur et les mêmes que Zadig a posées à l'ange. Le dialogue prend donc la forme d'un cercle parfait qui finit là où il a débuté. Voltaire nous laisse une question plutôt qu'une réponse. Zadig n'est pas l'histoire de la destinée. Le titre est trompeur. Zadig n'est sauvé ni par l'ange, ni par un hasard miraculeux, mais par ses amis et ses amies raisonnables. Zadig est l'histoire de l'être et du paraître. Le conte dit bien 'plus qu'il ne semble dire' (p. 1). Ce n'est pas un simple conte de fées; presque chaque phrase est chargée d'une double signification. Mais Zadig dit aussi moins qu'il ne semble dire, parce que le double sens est parfois faux sens, et que, lorsqu'il s'agit du hasard, il ne peut $\mathrm{y}$ avoir de réponse.

ROSEANN RUNTE

Université Sainte-Anne

Notes

1 Jacques Van den Heuvel, Voltaire dans ses contes (Paris: Armand Colin, 1967), pp. 170-79. Toute référence ultérieure à cette étude sera notée entre parenthèses dans le texte.

2 Roland Barthes, ed., Romans et contes de Voltaire (Paris, s.d.), p. 45.

3 François-Marie Arouet de Voltaire, 'Zadig,' Romans et contes, ed. H. Bénac (Paris: Garnier, 1960), p. 3. Toute référence ultérieure à cette édition sera notée dans le texte.

4 Voir, par exemple, Frederick M. Keener, 'Candide: Structure and Motivation,' Studies in Eighteenth-Century Culture, 9 (1979), 405-28; Roy S. Wolper, 'Zadig, a Grim Comedy?' Romanic Review, 64, 4 (novembre 1974), 237-48.

5 Nancy Senior, 'The Structure of Zadig,' Studies on Voltaire and the Eighteenth Century, 135 (1975), 135-41. Dans son introduction, Senior résume les arguments de V. Mylne, F.C. Green, N. Torrey et L. Crocker.

6 Voir, par exemple, E.J.H. Greene, 'The Destiny of Zadig,' Esprit Créateur, 3, 4 (hiver 1967), 243-51, et June Sigler Siegal, 'Voltaire, Zadig, and the Problem of Evil,' Romanic Review, 50 (1959), 29-32.

7 Voir George A. Perla, 'Zadig, Hero of the Absurd,' Studies on Voltaire and the Eighteenth Century, 143 (1975), 56-66; voir également Van den Heuvel, p. 172. 
8 René Pomeau, La Religion de Voltaire (Paris, 1956), p. 244; Lester G. Crocker, An Age of Crisis: Man and World in Eighteenth-Century French Thought (Baltimore: Johns Hopkins University Press, 1959), pp. 63-67.

9 Voir Virgil W. Topazio, Voltaire: A Critical Study of His Major Works (New York: Random House, 1967), pp. 34-35, pour une brève étude du rôle du 'mais' dans d'autres cuvres de Voltaire; voir aussi la communication non publiée de Timothy Scanlan, 'Le "Mais" dans Candide,' Atlantic Society for EighteenthCentury Studies Conference, Université de Moncton, 1978.

10 Frédéric Deloffre, Une Préciosité nouvelle: Marivaux ou le marivaudage (Paris: Armand Colin, 1967), passim.

11 Henri Bergson, 'Laughter,' dans Comedy, ed. Wylie Sypher (Garden City: Doubleday, 1956), p. 94.

12 Nous avons examiné ces sources dans une communication donnée au Congrès des Sociétés Savantes à Edmonton en 1981.

13 Il n'y a pas de preuves textuelles de sa souffrance. Cependant, lorsque je pose cette question à mes étudiants et à mes collègues, ils me répondent tous sans hésitation que Zadig se trompe comme d'habitude et qu'il devrait s'arrêter pour aider la deuxième demoiselle véritablement en détresse.

14 Jean Sareil, 'Le Comique par non-sens et faux sens dans les contes de Voltaire,' Studies in Eighteenth-Century Culture, 9 (1979), 482. 